\title{
MINISTERIO DE LAS CULTURAS: ANÁLISIS DE DISEÑO INSTITUCIONAL*
}

\author{
José Miguel Benavente \\ Jorge Larraín
}

\begin{abstract}
RESUMEN: El presente texto hace una revisión, desde el punto de vista de la economía de las instituciones, del proyecto de ley que propone crear un Ministerio de las Culturas, las Artes y el Patrimonio en Chile. En particular, se utiliza el enfoque de fallas de Estado para analizar cómo el proyecto que se discute actualmente en el Congreso Nacional aborda los problemas de inconsistencia dinámica, agencia y captura que afectan a distintos diseños de instituciones públicas, y en qué medida este proyecto constituye un avance respecto a la institucionalidad cultural existente en nuestro país. Si bien los autores comparten la idea matriz del proyecto de ley, de pasar a una orgánica de ministerio para asegurar una mejor inserción de la institucionalidad cultural en la estructura del Estado, advierten también una serie de
\end{abstract}

José Miguel Benavente. Doctor en economía por la Universidad de Oxford. Jefe de la División de Competitividad, Tecnología e Innovación del BID y profesor titular de la Escuela de Negocios de la Universidad Adolfo Ibáñez. Email: jm.benavente.h@gmail.com.

JoRge LARRAín. Máster en sociología y estudios culturales por la London School of Economics and Political Science. Ex coordinador de políticas públicas del Consejo Nacional de la Cultura y las Artes (2010-2014). Email: jlarrainm@gmail.com.

* Versión revisada de la conferencia expuesta por Benavente y Larraín en la segunda fecha del Simposio de Política Cultural organizado por Estudios Públicos, titulada "Desafíos en el diseño del Ministerio de las Culturas" y llevada a cabo el 27 de octubre de 2016 en el CEP. Los autores desean señalar: "Queremos agradecer los comentarios de Isabel Aninat, Ernesto Ayala, Rosanna Costa, Juan José Price, Juan Carlos Silva y a todos los participantes del seminario. Las apreciaciones y comentarios aquí planteados no representan necesariamente el sentir del directorio ni de la administración del Banco Interamericano de Desarrollo (BID). Los errores y omisiones son de exclusiva responsabilidad de los autores". 
elementos perfectibles, si es que se busca hacer del accionar público una tarea relevante y minimizar ineficiencias en el sector cultural. En línea con esto, se realizan propuestas concretas que buscan mejorar algunas de las falencias observadas.

PAlabras Clave: institucionalidad cultural, política cultural, economía de la cultura, fallas de Estado.

\title{
MINISTRY OF CULTURE: AN ANALYSIS OF THE INSTITUTIONAL DESIGN
}

\begin{abstract}
This article reviews the draft Bill that seeks to creaty a ministry of culture, arts and heritage in Chile from the viewpoint of the economics of institutions. It uses the government failure's approach to analyze how the draft Bill now being debated by the National Congress addresses the issues of dynamic inconsistency, agency dilemma and state capture that affect different designs of public institutions; and to what extent this represents progress compared to the institutional framework for culture now existing in our country. The authors agree with the main idea of the draft Bill of transferring responsibility to a ministry in order to ensure that culture becomes more a part of the government structure, but they also note several elements that could be improved if the intent is to make government action a main task and minimize inefficiencies in the cultural sector. Along these lines, they make concrete proposals intended to overcome some of the weaknesses found.
\end{abstract}

KEYWORDS: institutional framework for culture, cultural policy, cultural economics, government failures.

\section{INTRODUCCIÓN}

a discusión sobre el rol que debe jugar el Estado en el fomento y desarrollo de la cultura y las artes es de larga data. Momentos históricos de una fuerte evolución artística y cultural han contado con el apoyo y participación activa de estructuras políticas diversas que asumen funciones de Estado, aun en manifestaciones que antecedieron la formación y desarrollo de los sistemas democráticos. El rol de las ciudades-Estado italianas en el auge del arte renacentista, el impulso de edificaciones que hoy son patrimonio de la humanidad por parte de monarquías occidentales o el apoyo decidido que dieron las cortes 
vienesas a la evolución de la música son sólo ejemplos de una lista extensa.

La pregunta sobre la estructura institucional requerida para sostener distintas modalidades de intervención pública es más reciente. Ésta emergió al alero de - e influenciada por- el desarrollo de distintos regímenes de gobierno durante el siglo XX y de una burocracia profesional dedicada a la administración de los asuntos públicos. Este proceso ha estado marcado por concepciones ideológicas divergentes e interpretaciones sobre el rol del Estado coincidentes con dichas visiones y los valores públicos que éstas sostienen.

El surgimiento de las primeras instituciones culturales en Chile a fines del siglo XIX y su evolución y desarrollo durante el siglo XX han estado marcados por esta evolución de momentos históricos. No obstante lo anterior, es a partir de los años noventa que el debate público sobre institucionalidad cultural alcanzó el nivel de madurez y los consensos necesarios para generar instituciones de una relevancia mayor en la estructura política del Estado.

Es en este contexto en el que se sitúa la creación del Consejo Nacional de la Cultura y las Artes (CNCA) el año 2003 como órgano de dirección superior de las políticas culturales del país, y es en el marco de una razonable visión crítica sobre su evolución, desarrollo y problemas más importantes en el que se lleva a cabo la discusión actual en torno a la posibilidad de crear un Ministerio de las Culturas, las Artes y el Patrimonio en Chile.

Este texto realiza un análisis del proyecto de nueva institucionalidad cultural desde el punto de vista de las capacidades que una nueva arquitectura institucional pueda tener para avanzar en los objetivos de política pública que el país ha levantado para la cultura y las artes. Más específicamente, y tomando prestadas herramientas de la economía de las instituciones, buscamos evaluar el proyecto que actualmente se discute en el Congreso Nacional en términos de su preparación para enfrentar fallas de Estado recurrentes en instituciones públicas.

En la siguiente sección, y con el objetivo de demarcar lo que aquí denominamos como sector cultural, hacemos una breve reseña sobre los ámbitos económicos que serían susceptibles de la intervención del Estado. Adicionalmente, describimos resumidamente los principales hitos del desarrollo de las instituciones culturales en Chile, desde sus momentos fundacionales hasta el debate actual. 
En la sección 3, y en base a la literatura de la economía institucional, discutimos las características de las fallas de Estado más comunes en instituciones pertenecientes al sector público. Nos referimos aquí a los problemas de inconsistencia dinámica y agencia y a los riesgos de captura por parte de sectores beneficiados por las distintas políticas culturales. A continuación, describimos distintos arreglos de diseño institucional que buscan minimizar la ocurrencia de las problemáticas descritas.

En la sección 4 de este trabajo realizamos un análisis del texto del proyecto de ley (en adelante también el proyecto) que propone crear un Ministerio de las Culturas en Chile y hacemos propuestas que, a nuestro juicio, mejorarían el diseño institucional y lo harían más resiliente frente a las fallas de Estado aquí descritas.

Al realizar este trabajo nos asiste la convicción de que el Estado debe jugar un rol importante en el desarrollo de la cultura y las artes. En este sentido, coincidimos con muchas de las argumentaciones, tanto económicas como culturales, que justifican la intervención pública en el sector cultural. Sin embargo, consideramos fundamental contestar a la pregunta sobre cuán dotado está el Estado para lograr los objetivos públicos que se le plantean y para abordar, de manera eficiente, las distintas imperfecciones de mercado que se busca corregir. La elaboración de un buen diseño institucional resulta esencial para estos efectos.

Para terminar, una prevención. La mirada que proponemos es parcial. Los argumentos económicos no buscan reemplazar el debate público sectorial, sino aportar a éste y a la existencia de una pluralidad de discursos para analizar el mundo cultural.

Respecto al proyecto de ley, son muchos los aspectos destacables que reformulan temáticas importantes para nuestra política cultural. Entre éstos se puede mencionar la incorporación de representantes de nuestros pueblos originarios en los órganos colegiados del futuro Ministerio, la incorporación del patrimonio inmaterial cómo ámbito esencial de nuestras políticas sobre el patrimonio, y una concepción diversa de nuestras identidades culturales que toma en cuenta la realidad de un Chile mestizo y pluricultural.

No pretendemos discutir cada uno de estos objetivos, sino revisar aspectos del diseño de un futuro Ministerio que serán relevantes para alcanzar éstos y otros objetivos de política cultural. 


\section{ANTECEDENTES PRELIMINARES SOBRE EL SECTOR CULTURAL EN CHILE}

Antes de embarcarnos en la discusión sobre la institucionalidad cultural actual y futura en Chile, realizaremos una breve introducción sobre los ámbitos económicos y sociales que, para efectos de este trabajo, consideraremos como sector cultural. Una vez definido lo anterior revisaremos el rol que juega el sector público en el desarrollo, financiamiento y resguardo de estos sectores y la institucionalidad pública que se desprende de dicho rol. No es nuestra idea zanjar aquí un tema que lleva muchos años de debate, pero sí ayudar a acotar el ámbito sobre el cual se discute la institucionalidad chilena. Finalmente, realizaremos una breve reseña sobre la evolución histórica que ha tenido la institucionalidad nacional de apoyo al sector cultural.

\section{1. ¿De qué sectores estamos hablando?}

La discusión sobre los alcances y características del sector cultural tiene importancia para definir el rol que juega el Estado en su desarro1lo. Para Garretón et al. (2003), existen dos dimensiones de la cultura que dan origen a dos clases distintas de política cultural. Por un lado, existe aquella cultura que se define en un sentido amplio como "los modos de ser de una sociedad; sus visiones del pasado, del presente y del futuro"1, y en cuyo caso el rol del Estado es generar los espacios para que se lleve a cabo el debate público cultural en condiciones que reflejen la diversidad cultural de un país (ibídem). Esta acepción se asemeja a lo que se ha denominado como dimensión antropológica de la cultura. ${ }^{2}$

Por otro lado, la segunda dimensión se refiere a "las manifestaciones, aparatos y expresiones culturales como el patrimonio, los diversos campos de creación y difusión artística, las comunicaciones, las formas de cultura popular y las industrias culturales" (Garretón et al. 2003). Parte importante de la literatura sobre políticas culturales y sobre las

${ }^{1}$ Garretón et al. (2003) denominan "política cultural de base" a este tipo de política cultural.

2 David Throsby (2010) y otros. La definición de cultura que hace Unesco también se enmarca en la dimensión antropológica del término. 
formas institucionales que se requieren para implementarlas se refiere a esta última dimensión.

El alcance específico del sector cultural, en la segunda dimensión aquí descrita, ha sido objeto de debate tanto en la literatura especializada como en la práctica de las políticas culturales. Distintos países han adoptado distintas conceptualizaciones sobre el sector cultural. La discusión no se ha centrado en las actividades que parecen más naturalmente incorporadas al ámbito cultural, como pueden ser la danza, la música, las artes escénicas y las artes visuales. Ésta se ha dado en aquellas actividades o industrias que tiene un marcado sentido mercantil y que, aún teniendo características que justificarían el accionar del sector público en su apoyo, estarían más cerca del ámbito o quehacer de un ministerio de industrias, economía o producción que de una institución que promueva las artes y la cultura.

Para efectos de este texto, y siguiendo la caracterización económica planteada por David Throsby $(2008 ; 2010)^{3}$, consideraremos como sector cultural a las siguientes categorías de actividades. ${ }^{4}$ En primer lugar, aquellas que concentran una alta proporción de contenido cultural y bajo impacto económico, como son las artes visuales, escénicas (teatro, danza y ópera), la artesanía y la fotografía. En segundo lugar, aquellos sectores productivos que incluso teniendo un alto contenido cultural son capaces de producir bienes y servicios de manera industrializada. Este nivel contempla las industrias del libro, la música y el sector audiovisual. En un tercer nivel están aquellos sectores relacionados con la industria de los medios, como son la televisión, la radio y los medios escritos. Por último, situaremos en la periferia del sector cultural a aquellos sectores que transfieren contenido creativo y cultural a otras industrias, ya sea como demandantes o proveedores de bienes y servicios. Aquí se encuentran las industrias de la publicidad, la arquitectura, el diseño y la moda, entre otras.

Cabe señalar que cada una de estas industrias o sectores tienen diferentes contenidos culturales, como también difieren en su valor

${ }^{3}$ El economista australiano David Throsby conceptualizó el sector cultural a través del Modelo de Círculos Concéntricos $(2008 ; 2010)$. Éste clasifica los sectores culturales en función de la proporción de contenido o valor cultural de un determinado campo artístico y cultural respecto a su valor económico.

${ }^{4}$ Esta reinterpretación del Modelo de Círculos Concéntricos de Throsby fue utilizada por el CNCA en el Mapeo de las industrias creativas en Chile (CNCA 2014). 
económico y cultural (Benavente y Price 2011). De lo anterior se desprende, entre otras cosas, que el accionar público puede ser diferente en cada ámbito, pues hay diferencias en el tipo de bienes y servicios que se crean, la forma como se organiza su producción y los objetivos que persiguen quienes los producen.

En términos generales, la literatura sugiere que el accionar público no sólo se justifica por argumentos de carácter económico, como son la provisión de bienes públicos, la existencia de externalidades, valores de opción y valores de legado, entre otras situaciones, y donde el Estado busca solucionar fallas de mercado identificadas en algún campo cultural, sino también en base a argumentos puramente culturales. Para efectos de simplificar el análisis, se ha señalado que parte importante de los argumentos culturales pueden agruparse en dos categorías: el fomento de la excelencia e innovación en la producción artística y el fomento del acceso igualitario a la cultura (Throsby 2010).

En el marco aquí descrito, no es fácil dimensionar económicamente el sector cultural en Chile. Al debate sobre qué industrias o sectores económicos se tienen que considerar como parte de dicho sector, se debe añadir un nivel relativamente alto de informalidad en algunos campos artísticos y culturales, así como escasa información desagregada en fuentes tradicionales, como el Servicio de Impuestos Internos (SII) y las Cuentas Nacionales del Banco Central. No obstante lo anterior, se han hecho esfuerzos desde el $\mathrm{CNCA}^{5}$ que estiman el tamaño del sector cultural al año 2011 en 1,6 por ciento del PIB, con ventas agregadas en torno a los 11 mil millones de dólares y una relativamente baja presencia en el comercio exterior.

Respecto al gasto público cultural de Chile, no existen estimaciones precisas. La dispersión institucional — tema que discutiremos más adelante - se ve reflejada en las fuentes de información sobre gasto público. A los presupuestos de servicios del nivel central del Estado, como son el CNCA, la Dirección de Bibliotecas Archivos y Museos (Dibam) y la Dirección de Asuntos Culturales del Ministerio de Relaciones Exte-

${ }^{5}$ El más importante es la realización en 2014 del Mapeo de las industrias creativas en Chile (CNCA 2014), basado en metodologías similares realizadas en el Reino Unido y Australia. Éste considera una definición amplia de sector cultural e incluye sectores que pueden sobrestimar las mediciones, como la industria editorial periódica y la industria de medios informáticos. 
riores (Dirac), entre otros órganos, se les debe agregar el gasto público a nivel regional y municipal, y el apoyo fiscal indirecto que se genera por menor recaudación tributaria derivada de los beneficios de la Ley de Donaciones Culturales. En base a estas consideraciones y a partir de la Ley de Presupuesto de 2016, 'se puede estimar el gasto público en cultura en aproximadamente 315 millones de dólares, lo que representa el 0,55 por ciento del gasto público total de dicho año.

Tabla 1. GASTO DEL SECTOR PÚBLICO EN EL SECTOR CULTURAL DE CHILE AL AÑO $2016^{6}$

\begin{tabular}{lrrrc}
\hline & Miles de \$ & $\begin{array}{r}\text { Millones } \\
\text { de USD }\end{array}$ & $\begin{array}{c}\text { \% del } \\
\text { PIB }\end{array}$ & $\begin{array}{c}\text { \% del gasto } \\
\text { público }\end{array}$ \\
\hline CNCA_01 (Programas) & 84.645 .279 & 129.229 & & \\
CNCA_02 (Fondos de concursos) & 35.026 .923 & 53.476 & & \\
\hline CNCA & 119.672 .202 & 182.706 & 0,08 & 0,32 \\
\hline Dibam & 45.592 .755 & 69.607 & & \\
Red de Bibliotecas Públicas & 5.396 .086 & 8.238 & & \\
Consejo de Monumentos Nacionales & 2.790 .844 & 4.261 & & \\
\hline Dibam & 53.779 .685 & 82.106 & 0,03 & 0,14 \\
\hline CNCA + Dibam & 173.451 .887 & 264.812 & 0,11 & 0,46 \\
\hline Ley de Donaciones Culturales & 26.809 .000 & 41.889 & & \\
FNDR (2\% de Cultura) & 18.754 .273 & 29.304 & & \\
\hline Total Chile & 205.610 .660 & 315.060 & 0,13 & 0,55 \\
\hline
\end{tabular}

Fuente: Estimación de los autores en base a la Ley de Presupuestos de Chile del año 2016.

${ }^{6}$ La estimación consideró los presupuestos del CNCA y la Dibam (esta última incluye la red de bibliotecas públicas y el Consejo de Monumentos Nacionales), y un porcentaje del Fondo Nacional de Desarrollo Regional (FNDR) que habitualmente se asigna al sector cultural. Para este último dato se estimó el 2 por ciento que permite la glosa presupuestaria. Para el presupuesto que se asigna a la Ley de Donaciones Culturales, se asume el último registro disponible en el SII (2012) y se juzgó como gasto público el 50 por ciento de crédito tributario (el aporte público indirecto es mayor, dado que debe considerar el beneficio tributario de considerar como gasto el restante 50 por ciento). No se han considerado los presupuestos del Consejo Nacional de Televisión (CNTV) y programas vinculados al sector cultural de otros ministerios, como Educación, Obras Públicas y Relaciones Exteriores. 


\subsection{Desarrollo de la institucionalidad cultural en Chile}

Para abordar de mejor manera la discusión institucional actual, resulta conveniente describir sucintamente la evolución que ha tenido la institucionalidad de apoyo a la cultura y las artes en nuestro país. No pretendemos realizar un análisis histórico pormenorizado (hay excelentes textos abocados a este fin), ${ }^{7}$ sino entender el momento histórico en que se da la actual discusión y los cimientos sobre los que se construye.

Como hemos señalado, es posible encontrar una pluralidad de discursos que han sido utilizados históricamente para justificar la intervención del Estado en el campo cultural. Éstos abarcan desde lógicas político-simbólicas (Garretón et al. 2003) asociadas a la construcción de conceptos de "nación" y "cultura", la última en su dimensión más antropológica, hasta argumentaciones económicas que apuntan al rol que le cabe al Estado en corregir imperfecciones de mercado en el sector cultural.

En cualquier caso, el desarrollo de instituciones y políticas culturales en Chile ha estado marcado por los contextos político-discursivos de su historia.

Las primeras entidades públicas vinculadas al ámbito cultural tienen su origen en un momento fundacional de la nación chilena. El año 1813 se crea la Biblioteca Nacional; en 1843, la Universidad de Chile, y en 1857, el Teatro Municipal de Santiago. Todas instituciones con un rol importante en la construcción de una hegemonía lingüística e identitaria de Chile (CNCA 2012; Palominos 2014).

Ya en el contexto del Centenario de la República se creó el año 1925 el Consejo de Monumentos Nacionales, con la misión de "ejercer la protección y tuición del patrimonio cultural y natural de carácter monumental, velando por su identificación, protección oficial, supervisión, conservación y puesta en valor, potenciando su aporte a la identidad y al desarrollo humano" (Decreto Ley 651).

La Dirección de Bibliotecas Archivos y Museos (Dibam) fue creada en 1929, al alero del Ministerio de Educación, para administrar las organizaciones públicas más relevantes creadas hasta ese entonces en materia cultural. Éstas incluían la Biblioteca y Archivo Nacional, el Museo de Historia Natural de Santiago, la biblioteca Santiago Severin

${ }^{7}$ Ver Subercaseaux (2006), CNCA (2012), Palominos (2014) y Garretón et al. (2003), entre otros textos. 
de Valparaíso, el Museo Nacional de Bellas Artes y el Museo Histórico Nacional. En el transcurso del siglo XX, la Dibam se fue adaptando a las necesidades coyunturales derivadas del desarrollo cultural del país y progresivamente pasó a asumir responsabilidades sobre las políticas para el patrimonio cultural.

El largo período que transcurre entre el momento fundacional de la institucionalidad cultural chilena y las profundas modificaciones del Chile posterior a la dictadura está marcado por hitos importantes para el desarrollo cultural del país, como son la suscripción por parte de Chile de la Declaración Universal de los Derechos Humanos (1948), que introduce la concepción de derechos culturales en el debate público; el inicio de transmisiones de los canales de televisión universitarios y la creación en 1969 de Televisión Nacional de Chile; y la profundización de un modelo con mayor injerencia del Estado en el control de los medios de producción cultural bajo el gobierno de la Unidad Popular, proceso, este último, interrumpido por el golpe de Estado de 1973.

Desde el año 1990 en adelante, se posicionó con fuerza en el debate público la necesidad de renovar las instituciones culturales. Los primeros dos gobiernos de la transición constituyeron comisiones de expertos - las comisiones Garretón e Ivelic, respectivamente-, con el mandato de hacer un diagnóstico sobre el rol del Estado en el desarrollo cultural y de realizar propuestas de rediseño institucional. Este proceso de reflexión culminó con la creación, durante el gobierno del Presidente Lagos, del Consejo Nacional de la Cultura y las Artes (CNCA).

El año 2013, y con el objetivo de superar dispersiones en la estructura del Estado que persistieron a la creación del CNCA, se envió, por primera vez, un proyecto de ley para crear un ministerio de cultura en Chile, proyecto que fue reemplazado en 2016 con una indicación sustitutiva que propone la creación del llamado Ministerio de las Culturas, las Artes y el Patrimonio. Este último proyecto se encuentra actualmente en discusión en el Congreso Nacional.

\section{CONSIDERACIONES DE DISEÑO INSTITUCIONAL}

En la sección anterior enunciábamos la existencia de argumentos económicos y culturales que justifican el accionar público en el sector de la cultura y las artes. No obstante, dicho accionar no está exento de 
problemas, en especial aquéllos relacionados con un buen uso de los recursos públicos. Basados en la idea de fallas de Estado, discutiremos en esta sección cómo el accionar público (o la falta del mismo) podría generar ineficiencias y distorsiones económicas de una magnitud significativa. $^{8}$

En lo que sigue buscamos caracterizar las principales fallas de Estado que son aplicables en el contexto del apoyo público a las actividades culturales. Luego, y en base a dicha discusión, se plantea un conjunto de propuestas de diseño institucional que buscan reducir la manifestación de dichos problemas y sus consecuencias. En la última parte de esta sección describiremos en forma resumida algunos arreglos institucionales existentes hoy en el mundo y discutiremos cómo estos enfrentan las fallas de Estado aquí descritas.

\subsection{Fallas de Estado. Qué son y cuáles son sus implicancias}

La literatura económica institucional sugiere que pueden existir al menos tres tipos bien definidos de fallas de Estado. Cabe señalar que entenderemos por fallas de Estado aquellas situaciones en que el régimen de incentivos o el marco legal que regula la intervención pública en un determinado sector económico no es consistente con los objetivos que se buscan. Dicho de otra manera, las fallas de Estado conceptualizan situaciones donde no existe una congruencia entre el objetivo que se persigue con la participación pública y lo que las diferentes unidades mandatadas para ello efectivamente logran.

\subsubsection{Inconsistencia dinámica}

La falla de Estado más conocida y relevante a los efectos de la discusión de la política cultural se denomina inconsistencia dinámica. Este

${ }^{8}$ Aun existiendo buenos argumentos para que el Estado intervenga en un determinado sector o actividad, puede ocurrir que un inadecuado diseño institucional genere desarreglos de tal magnitud que el potencial daño producido por la intervención pública sea mayor que el problema que se buscaba resolver. A modo de ejemplo, en el contexto de la discusión sobre políticas industriales activas, el argumento de no intervención que se plasmó en el denominado Consenso de Washington (Williamson 1990) menciona que la mejor política industrial es que no exista política industrial alguna, ante la posibilidad de que los costos de intervención pudieran ser superiores a los beneficios potenciales. 
concepto apunta a aquellas situaciones en que, por las características de un determinado sector cultural, se requiere de un apoyo público consistente en el tiempo. Dada la naturaleza evolutiva de muchos de los desarrollos culturales, los apoyos actuales se van construyendo en base a las intervenciones pasadas. El problema asociado a esta falla surge cuando, por motivos que discutiremos, el esfuerzo de política pública cesa en el tiempo o bien cambia de objetivo en forma radical, en especial ante un cambio de la autoridad responsable de esas intervenciones.

Una de las principales características del apoyo a las actividades culturales es que los efectos buscados no se manifiestan en forma contemporánea, y las mejoras observadas en el tiempo no son fáciles de atribuir a los esfuerzos públicos realizados con anterioridad. Más aún, muchos de los apoyos públicos en el área cultural tienen una característica intangible. Ejemplos de este tipo son las políticas de educación artística y de formación de hábitos de participación cultural, las políticas industriales para el sector creativo y el conjunto de políticas regulatorias para sectores culturales específicos. Este tipo de intervenciones contrastan con otras de mayor tangibilidad, como pueden ser los proyectos de infraestructura cultural, que son visibles no sólo para los beneficiados directos, sino también para la comunidad como un todo.

La falta de tangibilidad de los resultados de algunas políticas culturales, sumada al hecho de que sus efectos pueden manifestarse sólo en el futuro, generan un potencial conflicto de intereses para aquellas autoridades que buscan resultados tangibles e inmediatos asociados a su gestión. En el margen, éstas podrían tener la inclinación de direccionar recursos públicos hacia actividades que les den mayor visibilidad, como un mecanismo de evaluación de su gestión.

El problema se genera cuando actividades que poseen estas características de poca tangibilidad y de impacto - a veces incierto - en el mediano plazo son relegadas a favor de otras de mayor inmediatez y tangibilidad, pero que son menos eficaces en lograr los objetivos de política pública planteados.

Una clara manifestación de este problema se observa en situaciones en que, ya sea por la llegada de nuevas autoridades o por el desinterés de las autoridades existentes, se decide modificar un mecanismo de apoyo público para el cual existe evidencia de un correcto diseño e implementación y, a pesar de lo anterior, no existe contrapeso alguno que 
pueda al menos elevar el costo político de dicha decisión. En este tipo de situaciones, y al realizar un análisis temporal de los objetivos y las propuestas de política pública que se proponen para alcanzarlos, no se observaría una consistencia adecuada en el tiempo. Y de allí el nombre de inconsistencia dinámica.

Este fenómeno es también observable en otras esferas del sector público, en particular, en instituciones relacionadas con el apoyo a las actividades científico-tecnológicas y, evidentemente, en el caso de las políticas para la educación en sus diferentes etapas.

\subsubsection{Problemas de agencia}

Una segunda falla de Estado que la literatura reconoce es aquella denominada como problema de agencia. La caracterización de este problema fue originalmente planteada por Williamson (1964) para el ámbito de las compañías. Esta falla describe situaciones en que, en contextos de juego estratégico y al existir una separación entre la propiedad (principal) y la administración (agente) de una compañía, el régimen de incentivos difiere para cada jugador, generando ineficiencias en el accionar de la firma. ${ }^{9}$ El clásico caso asociado a este problema se manifiesta cuando dos personas que están ligadas jerárquicamente en una institución tienen claras diferencias de intereses asociados a su labor y poseen diferentes grados de información sobre el efecto de sus decisiones.

La aplicación de este problema en el contexto de la institucionalidad pública se da en situaciones en que un ministro de Estado cumple el rol de principal, mientras que el jefe de una agencia que implementa políticas públicas, por ejemplo para el sector cultural, juega el rol de agente. Las funciones objetivo de ambos pueden ser muy diferentes y el director de la agencia (agente) suele tener mayor nivel de información que el ministro respecto del quehacer de la agencia, pudiéndose generar serios problemas de conflictos de objetivos e intereses o de agendas políticas.

${ }^{9}$ En particular, dada las diferencia de objetivos de maximizar el valor de la compañía versus el bienestar de la administración, el gerente de la empresa puede terminar tomando decisiones de asignación de recursos y esfuerzos que no están alineados con los intereses del director (o dueños) de la empresa. El problema central es que el agente posee más información que el principal sobre el quehacer diario de la compañía. 
Una manifestación del problema de agencia, que tiene particular relevancia en el sector público, es lo que se denomina problemas de coordinación. Si el diseño institucional no busca mecanismos para que los principales cuenten con mayor grado de información sobre lo que hacen los agentes o si no permite que puedan existir acciones correctivas por parte del principal, de manera de alinear los incentivos del agente con el principal, se pueden encontrar situaciones de ineficiencia, sobre posición de programas, descoordinación institucional y hasta competencia de diferentes agencias e instancias públicas para abordar ámbitos que requieren de la intervención del Estado.

\subsubsection{Riesgos de captura}

La tercera falla de Estado, y quizás una de las más complejas de solucionar cuando ya está presente, se denomina captura. Este problema alude a situaciones en que la agencia pública o un servidor que se ha vinculado en una relación de apoyo a determinados grupos de beneficiarios tiene dificultades para retirar dicha ayuda una vez que ésta ya no se justifica. Esta incapacidad de acción, motivada por potenciales represalias, amenazas de escándalo por parte de los beneficiarios o lisa y llanamente incompatibilidad legal para poder llevar adelante los cambios, configura aquella situación en que el Estado, representado por alguna de sus agencias, está capturado por grupos de interés.

En casos de captura, la pérdida de eficiencia económica se da cuando las fallas de mercado, u otras argumentaciones que hayan justificado el accionar público, han desaparecido o perdido su fuerza. Dado que los recursos públicos tienen usos alternativos, el dejar de asignarlos a estas actividades y direccionarlos a otras constituye una buena medida de política económica, y una clara muestra de ineficiencia si no se realiza.

Una de las grandes críticas que se hacen a programas de apoyo a la cultura y las artes radica en la quizá demasiada cercanía que existe entre los potenciales beneficiarios de dichos programas y los funcionarios o agencias que los diseñan. Más aún, puede ocurrir que una vez implementados los programas de apoyo al sector cultural, y en el caso de que éstos no funcionen eficazmente, ya sea por un diseño inadecuado o por no existir métricas que den cuenta de su impacto, sea muy difícil para la autoridad de turno eliminarlos o, al menos, cambiarlos. En estas 
situaciones se estarían generando ingresos extramarginales para los beneficiarios que son injustificados bajo el prisma de una buena política pública.

\subsection{Un buen diseño institucional como mecanismo para solucionar las fallas de Estado}

En lo que sigue, y a la luz de la descripción conceptual de las fallas de Estado, se propone un conjunto de soluciones genéricas asociadas al diseño institucional de apoyo público a las actividades culturales, orientadas a reducir o minimizar el impacto o pérdidas asociadas a ineficiencias resultantes de dichas fallas.

En primer lugar, y antes de sugerir las diversas formas en que se puede atacar el problema de inconsistencia dinámica, vale la pena distinguir tres tipos de acciones o políticas de apoyo público que pueden aplicarse en el ámbito de la cultura y las artes.

Existen, por una parte, aquellas propuestas de carácter estratégico, normalmente pensadas en el mediano y largo plazo y que buscan orientar los esfuerzos que el país necesita realizar para el sector cultural. En general, estas propuestas son recogidas en documentos de tipo estratégico y comúnmente elaborados en base a consejos o comités ad hoc, donde no sólo participan miembros del sector público sino también expertos en el área (académicos o investigadores) y representantes de los potenciales beneficiarios, los que buscan generar consensos sobre metas, objetivos generales y a veces métricas para hacer seguimiento a los objetivos planteados. Este tipo de documentos no sólo busca guiar el esfuerzo público, sino también las complementariedades con aquellos esfuerzos privados que puedan existir.

Enseguida, están las políticas propiamente tales, que dan cuenta de la voluntad e interés del poder ejecutivo de turno - no necesariamente del Estado-, las cuales se manifiestan en programas de gobierno o en planes de trabajo que se ejecutan durante el mandato de la autoridad responsable del sector.

Finalmente, están aquellas medidas operativas que de forma rutinaria se traducen en reglamentos, muchas veces derivados de proyectos de ley, que dan cuenta de los detalles administrativos de los programas de intervención en el sector. Estos últimos encarnan en términos prácticos (o deberían hacerlo) los intereses de la autoridad manifestados en el di- 
seño de política pública, y normalmente son ejecutados por las agencias debidamente creadas para ello.

En base a esta taxonomía, una forma directa y eficiente de evitar el problema de inconsistencia dinámica es la existencia de un consejo del más alto nivel compuesto por los participantes anteriormente sugeridos y que tenga entre sus objetivos: i) ser el responsable de la confección, revisión y actualización de una estrategia de mediano y largo plazo para el sector cultural; ii) velar, con la ayuda de un equipo técnico competente, por la consistencia entre las políticas públicas propuestas por el gobierno de turno y dicha estrategia, esto en base a evidencia, estudios de impacto y comparaciones internacionales, entre otros mecanismos; y iii) tener la autonomía y poder suficiente para explicitar, cuando la situación lo amerite, aquellas situaciones en que las propuestas de políticas de la autoridad se desacoplan de la estrategia de mediano plazo. Uno de los temas centrales de un consejo o comisión con estas atribuciones es que tenga un carácter permanente, con autonomía política del gobierno de turno así como financiera, e indiscutibles competencias técnicas y de representatividad en los temas que son de su competencia. Los miembros de dicho consejo podrán ser ratificados por las autoridades de un nuevo gobierno entrante o cambiar parcialmente su composición.

Con respecto a los problemas de agencia, uno de los principales síntomas de su existencia en el sector cultural se manifiesta en situaciones en que no hay claridad sobre la persona que posee la responsabilidad política de sacar adelante la agenda de políticas culturales. Un diseño institucional que permite visibilizar y canalizar en ojalá una sola autoridad dicha responsabilidad es una buena práctica que muchos países han seguido. De ser éste el caso, dicha autoridad deberá organizar al resto del aparato público (revisaremos algunos mecanismos viables) de tal manera de que no exista duda acerca de quién es el principal y quiénes los agentes, siguiendo la analogía con la literatura de organización industrial.

El principal problema de una configuración jerarquizada del aparato público se da cuando las actividades que están relacionadas con la promoción y apoyo público a la cultura y las artes no se circunscriben a una sola entidad superior de gobierno, como un ministerio o subsecretaría, extendiéndose la responsabilidad a otras agencias públicas. Esto 
sucede, por ejemplo, en el caso de las políticas de fomento a las industrias creativas que, teniendo una evidente relación con el sector cultural, dependen de otra instancia pública.

En estas situaciones, resulta conveniente instaurar mecanismos de comités interministeriales que ayuden a resolver potenciales problemas de coordinación. Éstos están generalmente organizados en un esquema de varios responsables de alto nivel liderados por uno solo que actúa como primus inter pares, quien es el responsable definitivo de la política pública para el sector. No es recomendable que otros agentes fuera del gobierno formen parte de estos comités ministeriales, aunque sí que lo hagan a nivel estratégico. Lo central, para dar cuenta del problema de agencia, es la visualización de un único responsable de dicha política, cuestión que debe quedar nítidamente plasmada en el diseño institucional respectivo.

Finalmente, el problema de captura puede ser resuelto de variadas maneras. Quizá la más directa y eficiente sea aquella en la que la entidad encargada de diseñar los diferentes instrumentos de apoyo público a la cultura y las artes no sea la misma agencia encargada de su implementación. En efecto, si los beneficiarios tienen la percepción de que son la misma entidad o muy cercanas en su quehacer y dependencia, el riesgo de captura aumentará, y una vez capturada la institución será muy difícil salir de dicha situación. Dicho de otra manera, si la agencia que diseña el instrumento no tiene alguna instancia de rendición de cuentas, que le haga un seguimiento, preferentemente basado en contratos de desempeño y respaldado en evidencia independiente, no tendrá ningún incentivo a modificar los programas y autoevaluarse, $y$, lo que es peor, no podrá eliminar algunos programas aunque genuinamente desee hacerlo por estimar que no logran el objetivo para el cual fueron diseñados. ${ }^{10}$

Una de las principales características que deben tener las agencias ejecutoras de políticas para el sector cultural es su relativa indepen-

${ }^{10}$ Si bien en Chile parte de esta labor es realizada por la Dirección de Presupuestos (Dipres), dependiente del Ministerio de Hacienda, esta entidad no tiene la suficiente capacidad para hacer un seguimiento detallado de cada programa en cada ministerio y goza también del problema de agencia, ya que no posee toda la información detallada acerca de la implementación del mismo y su función objetivo puede no coincidir con la del agente, en este caso, la agencia de implementación de la política. 
dencia del sistema público central, en especial político. Es deseable que los lineamientos de su quehacer vengan dados en forma explícita a través de un mandato de política pública emitido desde el estamento del cual dependa jerárquicamente. Adicionalmente, es aconsejable la constitución de un directorio que gobierne la respectiva agencia, cuyo presidente sea el representante de su mandante, y que albergue a otros participantes del ecosistema de la cultura y las artes, de manera de velar para que su accionar sea coherente con la política y, en consecuencia, con una estrategia nacional.

Existe evidencia sobre los efectos positivos que puede tener una relativa cercanía entre agencias ejecutoras de políticas públicas y sus respectivos beneficiarios, en especial en las etapas tempranas de la implementación de nuevas políticas (Sabel 2012). Ello, pues los ejecutivos de las agencias actúan como guías para los potenciales beneficiarios apoyándolos en procesos de postulación, adjudicación y seguimiento a programas públicos. No obstante lo anterior, esta relativa cercanía rigidiza la posibilidad de quitar los apoyos financieros cuando la decisión de hacerlo depende de ellos mismos. De allí la necesidad de contar con una entidad jerárquicamente superior, que decida la creación, diseño, modificación o cierre de programas culturales. ${ }^{11}$

\subsection{Casos de institucionalidad cultural y la forma en que éstos solucionan (o no) las fallas de Estado}

Antes de caracterizar los diferentes arreglos institucionales que han seguido varios países de interés, vale la pena introducir lo que entenderemos por institucionalidad cultural. Siguiendo a Squella (2008), institucionalidad se define como el conjunto de organismos de la administración del Estado - consejos, ministerios, agencias - que cumplen funciones en el ámbito de la cultura. Este mismo autor sugiere que dicha institucionalidad no es un medio en sí mismo, sino un mecanismo que posee el Estado para cumplir de mejor forma los deberes que tiene con el sector cultural.

${ }^{11}$ Es por eso que para esta instancia superior, típicamente un ministerio o subsecretaría, resulta contraproducente contar con un consejo asesor en el que puedan influir los beneficiarios en forma directa, capturando no sólo a la agencia —o agente-, sino también al principal. 
En términos conceptuales, y siguiendo a Chartrand y McCaughey (1989), los arreglos institucionales existentes hoy podrían agruparse en cuatro formas genéricas.

En primer lugar está la figura del Estado facilitador. Bajo esta forma, la institucionalidad adopta mecanismos de apoyo principalmente orientados a promover la participación del mundo privado en el financiamiento del sector cultural. Esto se logra a través de la implementación de políticas de apoyo fiscal indirecto en que el Estado otorga exenciones tributarias para individuos y corporaciones que financian proyectos u organizaciones culturales. En este tipo de institucionalidades, el desarrollo artístico y cultural depende principalmente del éxito comercial de los distintos proyectos y de su atractivo para donantes privados. Se ha señalado como una desventaja de sistemas que dependen de políticas de apoyo fiscal indirecto el ser vulnerables a ciclos económicos, pues el nivel de donaciones tiende a caer en ciclos económicos recesivos, interrumpiendo proyectos culturales de largo plazo y dando pocas garantías de consistencia temporal para una política de desarrollo del sector cultural. Quienes defienden este tipo de institucionalidades señalan como una de sus fortalezas el fomentar matrices diversas de financiamiento para el sector cultural y evitar riesgos de captura sectorial.

El caso paradigmático de Estado facilitador es Estados Unidos, donde el total del financiamiento público directo representa menos del cinco por ciento del financiamiento del sector cultural. El resto proviene de donaciones, que generan una menor recaudación tributaria para el Estado, y de ingresos privados de los agentes culturales. Entre las agencias públicas del Estado que sí entregan subsidios directos en Estados Unidos se incluye el National Endowment for the Arts - institución focalizada en la promoción de las artes, con un claro criterio de excelencia, y que asigna fondos en base a un sistema de evaluación de pares- y las distintas agencias estatales y locales de apoyo a la cultura.

El segundo tipo de institucionalidad cultural es la del Estado patrocinador. Bajo este modelo, el apoyo al desarrollo artístico y cultural se canaliza a través de consejos de artes. En estos casos, el gobierno determina el presupuesto global para apoyar el sector cultural, pero no interviene en forma directa en su asignación. El consejo (o consejos) tiene independencia del gobierno de turno y actúa como garante del proceso de asignación de fondos. El desarrollo artístico y cultural depende en este 
modelo del éxito comercial de un proyecto artístico, de su atractivo para donantes privados y de los fondos que entregue el consejo respectivo. El Reino Unido es el caso paradigmático de Estado patrocinador, pero este tipo de diseño institucional ha sido replicado en la mayoría de los países anglosajones (Australia, Nueva Zelandia y Canadá, entre otros).

Los consejos de artes, con participación del sector cultural y procesos de evaluación de pares para asignar fondos públicos, son susceptibles a riesgos de captura. En parte por este motivo, el Reino Unido, que instauró en los años 50 los sistemas de peer review (evaluación de pares), decidió posteriormente eliminar dicho método y reemplazarlo por revisiones internas del Arts Council, al considerar que los niveles de captura sectorial eran muy difíciles de controlar. No obstante lo anterior, los mecanismos de consejos han sido buenos sistemas para garantizar sostenibilidad en el tiempo de organizaciones culturales en estos países, como también han sido exitosos en fomentar la diversificación de fuentes de financiamiento de dichas organizaciones (cuestión que está en línea con dar sustentabilidad en el tiempo al sistema). Cabe señalar, finalmente, que la interacción entre Arts Council y las organizaciones culturales beneficiadas por sus fondos es regulada por un contrato de asignación de recursos que mide indicadores de desempeño, los que, de no ser cumplidos, pueden tener efectos sobre la siguiente ronda de financiamientos.

Finalmente tenemos la figura del Estado arquitecto $^{12}$. En este sistema, el apoyo público al desarrollo artístico y cultural se realiza a través de un Ministerio de Cultura principalmente con un criterio de bienestar social. El caso paradigmático es el francés. Si bien aquí existe una figura que es completamente responsable de la política cultural y artística del país, el ministro - con lo que se evita en parte problemas de agencia-, existen mayores espacios para la captura, ya que la institucionalidad aparece más integrada verticalmente. También pueden existir problemas de coordinación entre el nivel central y las oficinas regionales y el sector cultural en general. Una de las consecuencias de este tipo de arreglo institucional es la existencia de un bajo nivel de diversificación

12 Existe una cuarta categoría que se denomina Estado ingeniero, en que el Estado controla los medios de producción artística y cultural. Si bien fue una institución relevante en parte importante del siglo XX, particularmente en la Unión Soviética, ya no existe país alguno que la practique. 
de fuentes de financiamiento para la economía cultural, lo cual puede ser riesgoso para la continuidad de los proyectos y de las organizaciones culturales frente a restricciones de presupuestos públicos.

Chile tiene una combinación de elementos del Estado facilitador, patrocinador y arquitecto. El modelo vigente está fuertemente influenciado por la institucionalidad cultural mexicana, la que está actualmente en proceso de transformación hacia un ministerio.

Antes de entrar de lleno a la discusión sobre la institucionalidad que se sugiere en el nuevo proyecto de ley del ministerio, cabe mencionar que muchas de las ideas tanto conceptuales como aplicadas que se plantean en este ensayo ya se han discutido en otro contexto en Chile. En efecto, desde hace casi una década se viene analizando la posibilidad de crear un Ministerio de Ciencia, Tecnología e Innovación. Son muchas las similitudes, desde el punto de vista de la economía institucional, entre el ámbito de la cultura y las artes y el ámbito de la ciencia y la tecnología. No sólo existe un conjunto de actividades análogas a aquéllas propias de su ámbito más estricto, como son las ciencias básicas y las humanidades, que no responden necesariamente a incentivos económicos o de mercado, sino que también existen actividades que si bien se nutren de las anteriores, tales como la innovación y el emprendimiento de base tecnológica, pueden desarrollarse bajo un marco de incentivos de mercado. Las fallas de Estado que se han descrito en esta sección son aplicables al caso de la ciencia y la tecnología y se pueden manifestar con una similar intensidad. ${ }^{13}$

\section{ANÁLISIS DE DISEÑO INSTITUCIONAL AL PROYECTO DE LEY}

Esta sección analiza el proyecto de ley que propone crear el Ministerio de las Culturas, las Artes y el Patrimonio en Chile, ${ }^{14}$ desde la perspectiva de la economía institucional. En particular, nos centraremos

${ }^{13}$ Una discusión sobre los temas institucionales asociados a un Ministerio de Ciencia, Tecnología e Innovación para Chile puede encontrarse en CNIC (2007) y Comisión Asesora Presidencial (2013).

${ }^{14}$ Los comentarios y propuestas que realizamos en esta sección consideran el estado de tramitación en el Congreso Nacional del proyecto de ley al 16 de noviembre de 2016. A esta fecha, el proyecto ha sido aprobado por la Cámara de Diputados y está en discusión en la Comisión de Educación del Senado. 
en cómo el diseño institucional propuesto aborda $-\mathrm{O}$ no- las principales fallas de Estado descritas en la sección anterior: inconsistencia dinámica, problemas de agencia y riesgos de captura. Adicionalmente, con el fin de aportar al debate que se está desarrollando en el Congreso, realizaremos propuestas a algunos aspectos específicos del proyecto que a nuestro juicio no mejoran, o derechamente empeoran, el diseño institucional vigente en Chile.

\subsection{Inconsistencia dinámica}

Los riesgos para la política cultural asociados al problema de inconsistencia dinámica fueron considerados en el diseño del Consejo Nacional de la Cultura y las Artes, y a nuestro juicio fueron razonablemente bien resueltos.

La conceptualización del CNCA como un servicio público autónomo a cargo de formular e implementar políticas de Estado en materia cultural, entendidas éstas como "objetivos y principios que perduran en el tiempo más allá de un determinado gobierno" (Garretón et al. 2003), y cuya dirección superior recae en un órgano colegiado - el directorio nacional - con participación de la sociedad civil y la atribución de diseñar y aprobar dichas políticas, ha demostrado ser adecuada para alinear el esfuerzo cultural del Estado a un horizonte de largo plazo.

Las atribuciones que actualmente tiene el directorio nacional del CNCA permiten que éste incida en el proceso de confección de la política cultural y sobrepasan una dimensión meramente propositiva (Squella 2006). Dichas atribuciones se vieron reforzadas con una serie de disposiciones de la Ley 19.891 que facilitaron su autonomía frente a los gobiernos de turno.

En primer lugar, se estableció que los representantes del sector cultural en el directorio nacional y en los consejos regionales de cultura (en adelante también consejos regionales o CRCA) fueran designados por plazos predeterminados y que no pudieran ser removidos de sus cargos por la autoridad que los designa. ${ }^{15}$ En segundo lugar, se

${ }^{15}$ En el caso del directorio nacional, la designación es facultad de la Presidencia de la República, la que, además de nombrar a los tres ministros que representan al gobierno en dicho órgano colegiado, designa a cinco representantes de la sociedad civil (a propuesta de las organizaciones culturales del país). 
incorporaron instancias de control del Congreso Nacional y del propio directorio nacional a las designaciones que realiza el poder ejecutivo en los órganos colegiados del CNCA. El primero debe ratificar la propuesta de la Presidencia de la República de dos de los cinco miembros del directorio nacional que representan al sector cultural y el directorio nacional tiene la facultad de designar a cuatro de los siete miembros de los CRCA de una nómina de diez nombres propuestos por el intendente respectivo, previa consulta a las organizaciones culturales de la región (Ley 19.891, Art. 17).

Un tercer elemento para velar por la independencia de los órganos colegiados es la incorporación de personalidades designadas directamente por organizaciones autónomas respecto del gobierno. Éste es el caso del Consejo de Rectores de las Universidades Chilenas (CRUCh) $\mathrm{y}$ de los rectores de las universidades privadas autónomas que tienen derecho a designar a dos representantes en el directorio nacional del CNCA. En la misma línea se incorporó un galardonado con el Premio Nacional, elegido directamente por quienes han recibido esa distinción (Ley 19.891, Art. 5º).

Por último, el CNCA ha adoptado la práctica de designar a los representantes del sector cultural en el directorio nacional y en los CRCA de manera tal que el período de ejercicio de sus cargos esté traslapado a los períodos de gobierno. Estas designaciones se realizan a fines del segundo año de gobierno y tienen una duración de cuatro años. ${ }^{16}$ Como resultado de lo anterior, cada gobierno pasa la mitad de su período con un directorio nacional mayoritariamente designado por el gobierno anterior.

Este conjunto de disposiciones ha facilitado la continuidad de las políticas culturales en los primeros trece años de ejercicio del CNCA y explica, en parte, la mantención en el tiempo de estrategias y programas que requieren períodos de maduración relativamente largos. Desde luego, persisten iniciativas en que se privilegian intereses vinculados al ciclo político de cada gobierno, como lo demuestra la transversal tenden-

${ }^{16}$ Si bien no está contemplado explícitamente en la ley del CNCA, este procedimiento de designación es coincidente con el espíritu de dicho cuerpo legal, en términos de garantizar la existencia de políticas de Estado en materia cultural. 
cia a desarrollar obras emblemáticas de infraestructura cultural, ${ }^{17}$ pero incluso en estos casos la institucionalidad ha direccionado dichas iniciativas hacia los objetivos de política cultural previamente aprobados.

En síntesis, los órganos colegiados del CNCA han actuado consistentemente como un contrapeso efectivo a las autoridades de gobierno. El rol ejercido por el directorio nacional sobre el diseño y mantención de la política cultural, esto más allá de los alcances que debiera tener dicha política, y la autonomía de dicho órgano frente a las autoridades de gobierno son pilares fundamentales que sugerimos mantener en nuestra institucionalidad cultural.

El actual proyecto de ley (PL) que propone crear el Ministerio de las Culturas, las Artes y el Patrimonio modifica elementos importantes del diseño institucional vigente. El solo hecho de pasar a la figura de un ministerio limita el rango de atribuciones posibles que pueden tener los órganos colegiados dependientes de éste (cabe recordar que fueron éstas mismas limitaciones las que justificaron optar por la figura de un consejo y no de un ministerio el año 2003).

La más relevante de estas limitaciones es que en un ministerio la dirección superior del servicio debe recaer en la autoridad unipersonal, el ministro, en lugar de en un órgano colegiado. ${ }^{18}$ Para el caso que estamos abordando, el Ministro de las Culturas, las Artes y el Patrimonio tendrá a su cargo "la dirección superior de las políticas nacionales, planes y programas en materia de cultura y patrimonio" (PL, Art. 5), función que en la actualidad recae en el directorio nacional del CNCA.

${ }^{17}$ A modo de ejemplo, el Presidente Lagos gestionó los esfuerzos para la construcción del Centro Cultural La Moneda y el Centro Cultural Matucana 100. La Presidenta Bachelet, en su primer mandato, hizo lo mismo con el Centro Gabriela Mistral (GAM) y el Programa de Centros Culturales; y el Presidente Piñera propuso el Programa de Teatros Regionales y diversas iniciativas al alero del Programa Legado Bicentenario.

${ }^{18} \mathrm{Al}$ menos ésta ha sido la interpretación que tomó la actual administración del CNCA. Sin embargo, ésta es discutible y existen argumentos de destacados constitucionalistas (Soto Kloss 2013) que admiten un espacio para la existencia de órganos colegiados con funciones vinculantes sobre políticas públicas con dependencia administrativa de un ministerio. Un ejemplo de este tipo de órganos es el Consejo Superior de Educación, dependiente administrativamente del Ministerio de Educación, pero con atribuciones vinculantes sobre materias específicas de la política educacional. 
El futuro Consejo Nacional de las Culturas, las Artes y el Patrimonio (en adelante también CNCAP), sucesor del directorio nacional, será un órgano colegiado de veintiún miembros (en lugar de los once que tiene el directorio nacional actual), con integración mayoritaria de representantes del sector cultural y cuotas en su composición que asegurarían la presencia de consejeros provenientes de regiones distintas a la Metropolitana.

La propuesta de ampliar el órgano colegiado nacional del futuro ministerio refleja la intención de proveer de mayores grados de legitimidad social y cultural al CNCAP, a través de dar representación a sectores culturales anteriormente excluidos. Sin embargo, esta nueva estructura tiene el riesgo de complejizar y rigidizar en exceso el funcionamiento de este órgano colegiado. Es probable que un CNCAP del tamaño propuesto tenga dificultades importantes de coordinación interna y carezca de la agilidad y conocimiento técnico necesario para influir oportunamente en la agenda de políticas culturales. Si el objetivo es que el CNCAP se mantenga como un contrapeso real, su nueva estructura no lo facilitará.

En términos de las atribuciones del futuro CNCAP, el proyecto de ley establece tres niveles distintos de intervención sobre políticas culturales:

En un primer nivel, éste tendrá a su cargo aprobar la Estrategia Quinquenal Nacional de Cultura, entendida ésta como "las definiciones de objetivos estratégicos para el desarrollo cultural del conjunto del país" (PL, Art. 15). El CNCAP definirá el marco estratégico en el cual se diseñarán políticas públicas para el sector cultural. El proyecto asigna la responsabilidad de diseñar dicha estrategia al ministro, quien deberá presentarla para aprobación al CNCAP.

En un segundo nivel, el CNCAP intervendrá en el diseño e implementación de dos políticas públicas específicas: el Fondo Nacional para el Desarrollo Cultural y las Artes (Fondart) y el Fondo del Patrimonio Cultural. En estos casos, y continuando un rol que ya tiene el directorio nacional del CNCA, el CNCAP propondrá el diseño anual de cada concurso y las personas que cumplirán el rol de evaluación y selección de proyectos.

Por último, el CNCAP tendrá la función de representar el interés público en decisiones importantes del Estado en materia cultural, como 
son el proceso de designación de Premios Nacionales (rol que actualmente recae en el Ministerio de Educación) y la definición de manifestaciones patrimoniales que Chile postulará a la Lista Representativa de Patrimonio Inmaterial de la Humanidad de Unesco.

Respecto al primer nivel de atribuciones propuestas, parece razonable que el CNCAP sólo apruebe la Estrategia Quinquenal Nacional de Cultura y no las políticas y planes de trabajo anuales del futuro ministerio (como establece la actual Ley del CNCA). Esto es sincerar una situación de facto ya existente en los primeros trece años de ejercicio del CNCA.

El proyecto, aunque no de manera explícita, aclara una distinción importante para el funcionamiento de la institucionalidad cultural, la de políticas de Estado y políticas de gobierno, y asigna roles y entidades responsables de cada una de éstas. El órgano colegiado tendrá a su cargo la aprobación de políticas de Estado en materia cultural, asimilables en el actual proyecto de ley a la Estrategia Quinquenal Nacional de Cultura, en tanto construirá el marco estratégico de largo plazo dentro del cual se situarán las políticas y programas específicos de desarrollo cultural. A su vez, será la política de gobierno, de responsabilidad del ministro del ramo, la que diseñará e implementará políticas y programas específicos con miras a un horizonte de mediano plazo. Al revisar las dos políticas culturales aprobadas por el CNCA desde su creación (CNCA 2005; CNCA 2011), se puede observar que éstas sobrepasan el ámbito de políticas de Estado aquí descrito y abarcan una multiplicidad de iniciativas específicas que han resultado inabordables para las sucesivas administraciones del CNCA.

Ahora, para potenciar el rol que le cabrá al CNCAP sobre la política de Estado en materia cultural, se sugiere modificar sus atribuciones, de manera que éste asuma un rol activo en el diseño y preparación de la Estrategia Quinquenal Nacional de Cultura (en el actual texto sólo aprueba o rechaza la propuesta del ministro). Lo anterior es consistente con la incorporación en la ley de la figura de las Convenciones Nacionales de las Culturas, las Artes y el Patrimonio como instancias ampliadas de recepción de la opinión del sector cultural sobre dicha estrategia.

También resulta recomendable incluir entre las funciones del CNCAP el dar seguimiento a la política de gobierno con el objetivo de 
revisar la consistencia entre ésta y la Estrategia Quinquenal Nacional de Cultura. Para materializar esta función, el CNCAP debiera tomar conocimiento de las políticas, programas y presupuestos que se implementarán, de manera de dar una opinión pública sobre éstos en las Convenciones Nacionales. Si bien el poder ejecutivo sería soberano de considerar o no las opiniones del CNCAP, el proceso debiera ser obligatorio y generaría un contrapeso real a la formulación e implementación de políticas que haga el ministerio. ${ }^{19}$

Respecto al segundo nivel de intervención propuesto, parece poco razonable que el CNCAP mantenga atribuciones sobre el diseño e implementación de políticas públicas específicas. Esto sobrepasa el ámbito de políticas de Estado expuesto anteriormente y requiere de conocimiento técnico sobre los campos artísticos y culturales que abordan el Fondart y el Fondo del Patrimonio Cultural, cuestión que no es consistente con el hecho de que la nueva integración propuesta para el CNCAP privilegia la representatividad cultural de sus miembros por sobre su conocimiento técnico sectorial.

En este sentido, proponemos homologar el diseño institucional que ya ha sido adoptado para los sectores del libro y la lectura, música y audiovisual, y constituir órganos colegiados para las artes escénicas (teatro, danza y artes circenses) y las artes visuales (artes visuales, fotografía y artes mediales, entre otros campos) que actúen como agencias ejecutoras de la política cultural, participen del diseño anual del Fondart $\mathrm{y}$ formulen una política para sus respectivos sectores. ${ }^{20}$

El diseño e implementación del Fondo del Patrimonio Cultural debiera ser de cargo del ministerio, subsecretaría o Servicio Nacional del Patrimonio Cultural, al menos en una primera etapa. Más adelante se puede evaluar la posibilidad de dotar al Consejo de Monumentos Nacionales del carácter de órgano ejecutor de políticas para el patrimonio, lo que requeriría una modificación de la Ley de Monumentos Nacionales, cuya revisión excede los márgenes de este trabajo.

${ }^{19}$ El proceso para materializar este rol de "seguimiento" del CNCAP debiera quedar plasmado en un reglamento que apruebe dicho órgano colegiado.

${ }^{20}$ Esta propuesta requeriría de la apertura presupuestaria del Fondart en dos fondos paralelos, para las artes escénicas y las visuales. El remanente de recursos que actualmente se utilizan para el desarrollo de otros sectores (arquitectura, diseño, artesanías) o fines (líneas de asociatividad en regiones) debiera asignarse al presupuesto del futuro ministerio. 
Respecto al tercer nivel de atribuciones, esto es, la facultad que se le otorga al CNCAP para definir los mecanismos de designación para Premios Nacionales y otras decisiones relevantes para el Estado en materia cultural, opinamos que resultan razonables y constituyen un avance importante y valorado por el sector cultural.

En el plano de la autonomía de los órganos colegiados, el proyecto de ley propone modificaciones a los procedimientos de designación de los miembros del CNCAP y de los Consejos Regionales de las Culturas, las Artes y el Patrimonio (en adelante CRCAP o Consejos Regionales) que pueden requerir ajustes.

En el caso del CNCAP, los consejeros que representan al sector cultural pasarán a ser mayoritariamente designados por el ministro del ramo en base a propuestas de organizaciones de la sociedad civil pertenecientes al sector cultural. Se elimina, de esta manera, la ratificación del Congreso Nacional.

En la escala regional, el proyecto de ley hace una modificación menos drástica en la composición de los futuros CRCAP. Estos consejos incorporan representantes de los gobiernos regionales y municipalidades respectivas y mantienen la participación mayoritaria de representantes del sector cultural. Estos últimos pasan a ser designados por los seremis de Cultura (representantes del ministerio en la región). Se elimina aquí la facultad de designación de consejeros regionales que tenía el órgano colegiado nacional.

Por último, el proyecto establece una dieta de participación en todos los órganos colegiados del futuro ministerio (CNCAP, Consejos Regionales y consejos sectoriales de la música, libro y audiovisual).

Para efectos de resguardar la autonomía de los órganos colegiados, sugerimos que la función de designar a una parte de los representantes del sector cultural por parte de la Presidencia de la República, así como su ratificación en el Congreso Nacional, se mantenga. ${ }^{21}$

${ }^{21}$ El procedimiento propuesto en el proyecto de ley da la facultad de designación al ministro según una nómina de candidatos propuestos por organizaciones de la sociedad civil pertenecientes al sector cultural. Este procedimiento, que ya existe en el actual CNCA, genera incentivos para que el ministro de turno incida indirectamente para incluir en la nómina de candidatos a consejeros de su preferencia. 
Adicionalmente se sugiere que los representantes de las instituciones de educación superior y de los Premios Nacionales sean designados directamente según un procedimiento que el ministerio determine.

Sumado a esto, se propone incorporar explícitamente en el proyecto de ley el criterio de designación que ha adoptado el CNCA en sus trece años de existencia, según el cual los miembros del CNCAP y de los distintos Consejos Regionales, que no son designados por el poder ejecutivo en atención a sus cargos de gobierno, tengan períodos traslapados a los respectivos períodos de gobierno. ${ }^{22}$

Respecto a la escala regional, se consideran adecuadas las modificaciones propuestas a los Consejos Regionales. Sin embargo, y al igual como hemos señalado a nivel del CNCAP, se propone reincorporar la atribución existente en la institucionalidad actual, según la cual el órgano colegiado nacional aprueba las nóminas de representantes regionales del sector cultural propuestas por el poder ejecutivo.

\subsection{Problemas de agencia}

La principal falencia de la actual institucionalidad cultural tiene relación con problemas de agencia y, más específicamente, con los problemas de coordinación existentes entre las distintas agencias públicas que abordan el sector cultural.

Ésta no es una situación nueva. Ya la Comisión Garretón (Comisión Asesora Presidencial 1991) advertía que dos de las principales falencias de la organización del Estado en materia cultural eran la dispersión y duplicación de funciones en diversos órganos del Estado y la escasa coordinación existente entre éstos. Este diagnóstico fue reiterado por la Comisión Asesora Presidencial encabezada por Milan Ivelic (1997) y fue uno de los principales aspectos que buscó abordar el Consejo Nacional de la Cultura y las Artes. Como sostendremos, los avances del CNCA en esta materia han sido limitados, situación que contrasta con la efectividad con que se ha abordado el problema de inconsistencia dinámica ya descrito.

${ }^{22}$ El proyecto de ley establece que los procedimientos de designación de consejeros nacionales y regionales deberán definirse en un reglamento que tendrá que elaborar el ministerio. Para materializar la propuesta, el proyecto de ley debe indicar que el reglamento ha de prepararse de acuerdo con el criterio de designación sugerido. 
Al año 2003 (cuando se creó el CNCA), los organismos que tenían vinculación y presupuestos asociados al sector cultural eran: el Ministerio de Educación, a través de su División de Cultura, la Dibam y el Consejo de Monumentos Nacionales (además de otras áreas de menor relevancia); el Ministerio Secretaría General de Gobierno (Segegob), a través de su Departamento de Cultura; el Ministerio de Relaciones Exteriores, a través de la Dirección de Asuntos Culturales (Dirac), y el Ministerio de Obras Públicas, a través de su Oficina de Monumentos Nacionales y de su Dirección de Arquitectura. Estas agencias operaban de manera independiente, con poca coordinación entre sí y una casi inexistente articulación en la escala regional y municipal.

La creación del CNCA, como órgano de dirección superior en materia de políticas culturales, buscó superar la dispersión existente. Si bien la orgánica del CNCA contempló la absorción de una parte de las agencias pertenecientes al Ministerio de Educación (siendo las más importantes la División de Cultura y el Comité Calificador de Donaciones Privadas) y de la totalidad de los órganos y funciones de la Segegob, se mantuvo, por razones que expondremos más adelante, a la Dibam y al Consejo de Monumentos Nacionales al alero del Ministerio de Educación. Adicionalmente, se optó por mantener a la Dirac como un órgano dedicado a la política internacional en el Ministerio de Relaciones Exteriores. ${ }^{23}$

Con los objetivos de integrar los ámbitos propios de la Dibam, el Consejo de Monumentos Nacionales (CMN) y la Dirac en la nueva institucionalidad cultural y de evitar que se reprodujera la fragmentación y superposición de políticas existentes hasta ese entonces, se incluyeron como funciones del CNCA, según la Ley 19.891, el diseñar políticas para el patrimonio y al ámbito internacional de nuestras artes (Art. $3^{\circ}$, números 1, 2, 4, 7 y 11). A su vez, se incorporaron al directorio nacional del CNCA los ministros de Educación y de Relaciones Exteriores (o sus respectivos representantes) y los seremis de Educación en cada Consejo Regional de Cultura. Por último, la ley le entregó al CNCA el mandato de coordinación de todas aquellas reparticiones públicas que cumplieran funciones en el ámbito de la cultura (Ley 19.891, Art. $3^{\circ}$, n. $^{\circ}$ 9).

En el mismo contexto de velar por la coordinación entre instituciones públicas, las leyes que en los años previos y posteriores a 2003

${ }^{23}$ La figura 1 en el Anexo, muestra las instituciones públicas que actualmente tienen vinculación con políticas para el sector cultural. 
crearon los consejos sectoriales del libro y la lectura, música y audiovisual también incorporaron en dichos consejos a representantes de agencias técnicas vinculadas al campo cultural de los ministerios de Educación, Economía y Relaciones Exteriores. ${ }^{24}$

A pesar de estos arreglos institucionales, el CNCA, en sus primeros trece años de funcionamiento, no ha logrado consolidar su mandato de coordinación sobre las políticas culturales del país.

En el ámbito patrimonial es donde más nítidamente han persistido las dispersiones y duplicidades advertidas a principios de los noventa. La Ley 19.891 intentó resolver esta situación a través de facultar al Presidente de la República para dictar un decreto que regulara la relación que tendrían la Dibam y el CMN con el CNCA (Disposiciones Transitorias Art. $1^{\circ}$ ). Se buscaba, por esta vía, construir un mecanismo formal de integración de estas instituciones. No obstante lo anterior, la fuerte oposición interna de la Dibam hizo imposible la materialización de este decreto.

Desde ese momento la coordinación entre la Dibam y el CNCA ha sido mínima. La autonomía presupuestaria de ambas instituciones, la coexistencia de atribuciones sobre el ámbito patrimonial en dos órganos del mismo nivel jerárquico-administrativo y la existencia de culturas organizacionales muy distintas han marcado una relación conflictiva en todos los gobiernos desde el año 2003 a la fecha. Existen numerosos ejemplos que permiten observar el efecto que esta "distancia" institucional ha producido sobre la efectividad de políticas públicas en los ámbitos del patrimonio y la lectura. ${ }^{25}$

${ }^{24} \mathrm{Al}$ Consejo del Libro se incorporó el subdirector de bibliotecas de la Dibam y un representante del Mineduc; al Consejo Audiovisual, un representante de ProChile (RR.EE.), uno de la Corfo y un representante del Consejo Nacional de Televisión. El Consejo de la Música, por su parte, incorporó un representante de la Presidencia de la República y de la Asociación Chilena de Municipalidades.

${ }^{25}$ Cuatro casos que pueden ilustrar los efectos descritos son: i) política de infraestructura cultural en regiones con baja coordinación entre proyectos del CNCA (que ha llevado adelante el Programa de Centros Culturales) y la Dibam (que ha desarrollado museos y bibliotecas públicas en las regiones del país); ii) brecha creciente entre el estado de los museos nacionales (dependientes de la Dibam) y las organizaciones culturales nacionales desarrolladas desde el CNCA (por ejemplo, GAM, Centro Cultural La Moneda o más recientemente el Centro de las Artes de Cerrillos); iii) Programa de Adquisiciones de Libros del Consejo del Libro con baja interacción con el sistema de bibliotecas públicas; y iv) Fondo del Patrimonio Cultural del CNCA con poca influencia de los órganos técnicos de la Dibam y el CMN. 
Otro ámbito importante en que el rol de coordinación interinstitucional ha sido poco efectivo es en materias de educación artística. El CNCA no ha logrado tener influencia frente al Ministerio de Educación para cumplir con su función de "dar suficiente expresión a los componentes culturales y artísticos en los planes y programas de estudio y en la labor pedagógica y formativa de los docentes y establecimientos educacionales" (Ley 19.891, Art. $3^{\circ}$, n. $^{\circ}$ 5). Una muestra de esta falta de relevancia son los sucesivos cambios en el currículo de educación básica que han disminuido las horas de educación artística y la precaria evolución que ha tenido el programa de escuelas artísticas del Ministerio de Educación.

Quizás una excepción a los ámbitos descritos es el de las políticas de internacionalización de nuestras artes donde, después de años de baja interacción con otras entidades de gobierno (principalmente dependientes del Ministerio de Relaciones Exteriores), ha habido avances relevantes de trabajo interinstitucional en torno a proyectos considerados estratégicos para Chile, ${ }^{26}$ que han sido canalizados a través de un comité interministerial con el Ministerio de Relaciones Exteriores. Otro ejemplo más reciente de coordinación institucional es la formación de un comité interministerial de fomento de la economía creativa, iniciativa de la actual administración del CNCA, con el objetivo de articular de forma coherente los distintos instrumentos existentes para el fomento del sector creativo.

Es posible argumentar que el bajo nivel de coordinación interinstitucional existente tiene relación con la conceptualización del CNCA como una organización colegiada sin rango ministerial, en una cultura administrativa - la chilena - altamente vertical y jerarquizada. La figura de un consejo no ha logrado el peso específico ni la transversalidad necesaria para generar políticas de Estado en materia cultural. Este solo argumento de diseño institucional parece ser suficiente para justificar la creación de un ministerio. De hecho, ha sido esgrimido tanto en el proyecto de ley que envió el gobierno del Presidente Piñera como en la actual indicación sustitutiva a dicho proyecto que está en discusión en el Congreso.

${ }^{26}$ Un ejemplo es el trabajo coordinado que hace el CNCA, la Dirac, ProChile y la Fundación Imagen de Chile (las tres últimas dependientes del Ministerio de RR.EE.) para los envíos a la Bienal de Venecia de Artes y Arquitectura y el proyecto de Marca Sectorial Cinema Chile, donde participan ProChile y el Consejo Audiovisual, para posicionar el cine chileno en el mundo. 
Existe consenso sobre la necesidad de que la institucionalidad cultural se estructure en torno a la figura de un ministerio. Las atribuciones de carácter horizontal del CNCA, esto es sobre ámbitos que están fuera de su línea jerárquica, no han dado resultado y se han transformado en meros enunciados. Adicionalmente, la representación del Ministerio de Educación y el Ministerio de Relaciones Exteriores en el CNCA no han sido condición suficiente para asegurar el enforcement de políticas culturales sobre temas de educación, patrimonio e internacionalización.

La definición del proyecto de ley respecto de que será el ministro quien ejercerá la dirección superior del futuro ministerio (en el CNCA es el órgano colegiado el que ejerce dicho rol), no obstante ser discutible desde el punto de vista de la autonomía e influencia que tendrán los órganos colegiados, establece de manera clara la responsabilidad sobre la política cultural y mejora las posibilidades de una buena coordinación vertical sobre órganos y ámbitos que pasarán a la estructura jerárquica del ministerio (Dibam y CMN), y horizontal sobre órganos que se mantendrán fuera de la estructura del ministerio, como el Ministerio de Educación, la Dirac del Ministerio de Relaciones Exteriores, el Consejo Nacional de Televisión y la Corfo, entre los más relevantes.

También parece razonable la mantención de los ministros de Educación y Relaciones Exteriores y la incorporación del ministro de Economía (o quien éste decida que lo represente) en el CNCAP. A su vez, el mayor peso que tendrán los consejeros pertenecientes al sector patrimonial propenderá a recuperar la importancia relativa de las políticas para el patrimonio en las políticas culturales.

No obstante lo anterior, existen aspectos de la estructura orgánica planteada en el proyecto de ley para el nuevo ministerio, así como de las funciones propuestas para éste, que ponen en riesgo la existencia de una coordinación eficaz en sus dos modalidades — horizontal y vertical- en ámbitos importantes de la política cultural.

Preocupa particularmente la incidencia que tendrán las futuras políticas culturales sobre temas de educación. En la definición de objetivos del nuevo ministerio se limita su rol en el campo educacional, circunscribiéndolo al "fomento de la educación artística no formal como factor social de desarrollo" (Art. $1^{\circ}$, n. $^{\circ} 8$ ) y lo excluye de intervenir en el sistema formal de educación. Esto es preocupante si se considera 
que existe amplia evidencia sobre la importancia de la exposición temprana a la cultura y las artes en la educación formal para mejorar los niveles de participación cultural (Robinson et al. 1985; Schuster 1991; O'Hagan 1998; Throsby 2010; Price 2011), uno de los objetivos centrales del futuro ministerio.

Se sugiere, en consecuencia, agregar entre las funciones del ministerio el "participar, en coordinación con el Ministerio de Educación, en diseñar políticas, planes y programas destinados al fomento de las artes, de la lectura y del patrimonio cultural del país en la educación formal, en todos sus niveles", función que estaba presente en el proyecto de ley del año 2013 y que ya había sido aprobada por la Comisión de Cultura de la Cámara de Diputados. Una manera específica en que estas iniciativas se pueden materializar es a través de convenios de desempeño que regulen la ejecución de programas en la órbita del Ministerio de Educación con fondos del presupuesto del futuro Ministerio de las Culturas. ${ }^{27}$

Otro aspecto importante que no aborda el proyecto es la coordinación necesaria para el diseño e implementación de políticas de fomento a la lectura entre el Ministerio de Educación, ${ }^{28}$ el Consejo Nacional del Libro y la Lectura y la actual Dibam. El proyecto de ley mantiene al Sistema de Bibliotecas Públicas — actualmente parte de la Dibam - en la orgánica del futuro Servicio del Patrimonio Cultural. Con el objetivo de dar mayor peso a la implementación de la recientemente aprobada Política Nacional de Fomento al Libro y la Lectura y de establecer una plataforma más simple y ágil de coordinación con el Ministerio de Educación, se propone localizar el Sistema de Bibliotecas Públicas en la orgánica del Consejo del Libro. ${ }^{29}$

${ }^{27}$ Tal como lo hace, por ejemplo, la División de Innovación del Ministerio de Economía con la Corfo y Conicyt. En este caso, la primera es responsable de la administración del Fondo de Innovación para la Competitividad (FIC), resultante del impuesto específico a la minería (royalty minero), y delega su ejecución tanto en Conicyt como en la Corfo en base a contratos de desempeño.

${ }^{28}$ El Ministerio de Educación contiene las bibliotecas CRA (Centro de Recursos para el Aprendizaje), quizás el esfuerzo más consistente realizado por el Estado para el fomento de la lectura en Chile.

${ }^{29}$ El presupuesto de la red de bibliotecas públicas ya es independiente del de la Dibam, lo que facilitaría la implementación de esta propuesta. Puede ser razonable que la Biblioteca Nacional, dada su historia e importancia patrimonial, se mantenga al alero del Servicio Nacional del Patrimonio. Desde luego, el Archivo Nacional y el Sistema Nacional de Archivos deben mantenerse en el Servicio Nacional del Patrimonio. 
En cuanto a la estructura organizacional que propone el proyecto de ley para el patrimonio cultural, ésta resulta excesivamente compleja y puede tender a dificultar la coordinación vertical necesaria para implementar y evaluar las políticas para el patrimonio cultural que el nuevo ministerio lleve adelante. ${ }^{30}$ No se entrega argumentación que justifique la existencia de una Subsecretaría del Patrimonio y de un Servicio del Patrimonio Cultural separados. Este último servicio tendrá a su cargo tres "sistemas nacionales": el de museos, archivos y bibliotecas públicas, cada uno con un consejo asesor.

La orgánica propuesta para el sector patrimonial contrasta con la que se propone para las artes e industrias culturales. Ésta sólo contempla una Subsecretaría de las Artes, Industrias Culturales y Culturas Populares que reemplaza al actual CNCA y coordina los tres consejos sectoriales (música, libro y audiovisual). Adicionalmente, tiene a su cargo la dirección administrativa de las secretarías regionales ministeriales y la administración y servicio interno del ministerio.

Con el objetivo de simplificar la estructura del nuevo ministerio y generar una coordinación eficaz entre principal (en este caso, el ministro) y la agencia que tenga a su cargo ejecutar las políticas para al patrimonio, proponemos constituir una Subsecretaría del Patrimonio que absorba la orgánica, funciones y atribuciones del Servicio del Patrimonio Cultural y coordine los sistemas de museos y archivos, y también la secretaría técnica del Consejo de Monumentos Nacionales. ${ }^{31}$

Desde el punto de vista de la estructura regional del ministerio, la modificación que se propone a la composición de los Consejos Regionales de Cultura parece adecuada para efectos de mejorar la coordinación horizontal en las escalas regional y municipal. Se incorpora un representante de las municipalidades y el gobierno regional respectivo y se mantiene la presencia del seremi de Educación. La presencia de los órganos regionales de los ministerios vinculados al quehacer cultural, complementada con una integración mayoritaria de representantes del sector cultural regional apuntan a generar un ecosistema institucional robusto y cohesionado en las regiones de nuestro país.

Para ser consistente con lo propuesto para la Subsecretaría del Patrimonio Cultural, las secretarías regionales ministeriales de las Cul-

${ }^{30}$ Ver figura 2 en Anexo.

${ }^{31}$ Ver figura 3 en Anexo. 
turas, las Artes y el Patrimonio debiesen absorber la orgánica, atribuciones y funciones de las Direcciones Regionales del Patrimonio (órganos de desconcentración ${ }^{32}$ del Servicio del Patrimonio Cultural). Dichas secretarías serían coordinadas por la Subsecretaría de las Artes, Industrias Culturales y Culturas Populares en términos administrativos y por cada subsecretaría en ámbitos de su respectiva competencia.

\subsection{Riesgos de captura}

Los riesgos de captura son inherentes a las políticas públicas que abordan el sector cultural. Ya sea que el objetivo de la política sea reducir desigualdades de acceso a bienes y servicios culturales, corregir fallas de mercado en algún sector o industria cultural específico o aumentar la demanda agregada de un bien considerado meritorio, ésta intervendrá en sectores en los que necesariamente confluirán múltiples intereses. En este contexto, será difícil para la agencia pública redireccionar recursos a usos alternativos una vez iniciada una política o programa.

El diseño colegiado que adoptó el CNCA fue, en parte, determinado por la pre-existencia de una forma específica de política cultural: la concursabilidad de fondos públicos para la creación y el desarrollo de la cultura y las artes.

Ya el año 1992 se había creado el Fondo Nacional para el Desarrollo Cultural y de las Artes (Fondart), al alero de la Ley 19.891, y el año 1993, el Fondo Nacional de Fomento del Libro y la Lectura, con su respectiva Ley 19.227. En ambos casos se optó por una lógica de concursabilidad de fondos públicos y de evaluación de pares para determinar los proyectos beneficiados. Posterior a la creación del CNCA, pero en la misma lógica del Fondart y del Fondo del Libro, se crearon el año 2004 el Fondo de Fomento Audiovisual y el Fondo para el Fomento de la Música Nacional.

Los tres fondos de las denominadas "industrias culturales" pasaron a ser gobernados por órganos colegiados con representación de los

${ }^{32}$ Se habla de desconcentración, ya que estas direcciones regionales no tendrán poder resolutivo de todos los proyectos, inversiones y otros tipos de ejecuciones presupuestarias que involucren a agentes de la región. Si fuera así estaríamos hablando de descentralización. 
sectores culturales respectivos. Así se crearon el Consejo Nacional del Libro y la Lectura, el Consejo de Fomento de la Música Nacional y el Consejo del Arte y la Industria Audiovisual. La composición de los consejos fue definida en las respectivas leyes.

Para el caso del Fondart, y ante la inexistencia de un consejo ad hoc, se determinó que el órgano de gobierno sería el directorio nacional del CNCA. De la misma manera, el Fondo del Patrimonio Cultural, creado por glosa presupuestaria el año 2012, ha sido coordinado por el CNCA y pasaría a depender, según propone el actual proyecto de ley, de la futura agencia patrimonial con supervisión del CNCAP.

Las disposiciones que actualmente considera la legislación para atenuar potenciales riesgo de captura son: i) el rol de los consejeros pertenecientes a cada órgano colegiado está definido en términos de su representación sectorial y no de las organizaciones que los proponen o designan, ${ }^{33}$ ii) el rol "tácito" de velar por el interés público que ejerce el ministro de Cultura, quien preside los tres consejos sectoriales, y iii) las disposiciones de conflicto de interés transversales al sector público que entrega la Contraloría General de la República y la Ley de Bases para la Administración del Estado.

Se hace necesario analizar el grado de captura sectorial que han tenido los cuatro fondos para el sector cultural y la capacidad que ha tenido la institucionalidad cultural para resguardar el interés público frente a intereses corporativos que pudiesen expresarse en los consejos sectoriales, ${ }^{34}$ en el contexto de que dichos consejos diseñan, implementan $\mathrm{y}$, en algunos casos, asignan fondos públicos para sus respectivos

${ }^{33}$ Son muchos los casos en que quienes designan a los miembros de consejos sectoriales son organizaciones que representan intereses corporativos en el respectivo sector. Sólo a modo de ejemplo, en el Consejo del Arte y la Industria Audiovisual se eligen representantes de los productores audiovisuales (asociados en la APCT), los productores y directores de documentales y de asociaciones de guionistas, entre otras.

${ }^{34}$ Existen formas para analizar la existencia (o no) de este problema. Por ejemplo, se puede estudiar en qué medida las asignaciones presupuestarias (particularmente a nivel de los tres fondos sectoriales) coinciden con las prioridades de la estrategia y política sectorial o, más bien, son coincidentes con intereses corporativos que están representados en el respectivo consejo (caso este último que sería sintomático de una situación de captura). Alternativamente, se puede analizar si han existido evaluaciones de resultados y de impacto a los programas que hayan generado cambios en los criterios o lisa y llanamente el cierre de programas no justificables. 
sectores. Este análisis sobrepasa los alcances del presente trabajo, pero resulta prioritario para la consolidación de la institucionalidad propuesta.

Resulta preocupante, al mismo tiempo, la baja presencia que ha tenido esta particular falla de Estado en el debate público. Tanto el largo debate que precedió la creación del CNCA como las discusiones en torno a los proyectos de ley propuestos para modificarlo han omitido los riesgos de captura existentes en dichos diseños institucionales. En el caso del actual proyecto de ley, parte de la explicación para esta omisión tiene que ver con que éste busca modificar la estructura superior de nivel nacional y regional de la institucionalidad cultural, y no sus agencias sectoriales (consejos del libro, la música y audiovisual y Consejo de Monumentos Nacionales para mencionar los más importantes).

Respecto a aspectos que sí aborda el proyecto de ley, podemos señalar que nos parece de suma importancia que el ministro mantenga el rol de presidente en cada uno de los consejos sectoriales (con facultad de delegar su representación si así lo requiere). En el actual proyecto es el subsecretario de las artes e industrias culturales el que asume el rol de presidente en los tres consejos de industrias culturales y el director nacional del Servicio del Patrimonio Cultural preside el Consejo de Monumentos Nacionales.

Adicionalmente, parece necesario revisar las funciones y atribuciones de las agencias ejecutoras de políticas culturales (contenidas en las leyes que regulan los tres consejos sectoriales). En términos conceptuales, éstas (las agencias) no pueden tener la responsabilidad de modificar o eliminar programas que han dejado de ser efectivos como política pública, pues enfrentan un claro conflicto de interés debido a su cercanía con los beneficiarios de los mismos programas. Este rol debe recaer en un órgano jerárquicamente superior, en este caso el ministerio, que vele por el interés público y asuma la responsabilidad de priorizar los presupuestos de acuerdo a los lineamientos de la estrategia y política nacional respectiva.

\section{CONCLUSIONES}

En este texto hemos hecho una revisión, desde el prisma de la economía institucional, del proyecto de ley que propone crear un Ministerio de las Culturas, las Artes y el Patrimonio en Chile. 
Sostenemos que la idea matriz del proyecto, de consolidar las diversas organizaciones culturales existentes - o al menos las de mayor relevancia - en un ministerio que pueda implementar eficazmente una política de Estado en materia cultural, es adecuada. A trece años de la creación del Consejo Nacional de la Cultura y las Artes, es razonable reconocer que su rol de coordinación sobre órganos de igual jerarquía institucional ha sido limitado.

No obstante lo anterior, consideramos conveniente hacer modificaciones que resguarden la autonomía de los órganos colegiados frente al poder ejecutivo y mantengan su rol como garante de políticas de Estado orientadas al largo plazo. Adicionalmente, parece de vital importancia que se revise la orgánica propuesta para la Subsecretaría del Patrimonio Cultural. Si ya la institucionalidad de 2003 fue ineficaz en minimizar la ocurrencia de problemas de agencia, y en particular de coordinación, respecto a las políticas sobre el patrimonio, sería inexplicable que una estructura excesivamente compleja mantuviera los mismos problemas trece años después.

Respecto a riesgos de captura, parece conveniente hacer una revisión sobre el nivel de captura sectorial que pueda existir en los consejos sectoriales del libro, la música y lo audiovisual, y sobre todo proponer ajustes a la estructura de diseño e implementación de políticas y programas de apoyo a dichos sectores, de manera de poder ajustar programas que no se justifiquen y redireccionar recursos hacia usos más prioritarios.

En la introducción a este trabajo, y junto con delimitar sus alcances y objetivos, enunciamos elementos destacables del proyecto de ley sobre los cuales no nos referiríamos. Parece necesario señalar que también existen vacíos importantes en el proyecto. Algunos de estos últimos se explican en la decisión justificada de no modificar varios cuerpos legales que regulan el sector cultural a la vez. ${ }^{35}$ Pero otros resultan menos comprensibles. En este sentido, vale la pena preguntarse: ¿Es suficiente la apuesta descentralizadora del futuro ministerio? ¿Es razonable que el Ministerio de las Culturas no tenga un rol relevante sobre la televisión pública cultural? ¿Puede una política de participa-

${ }^{35}$ Nos referimos a la Ley de Monumentos Nacionales y a las leyes que regulan el Fondart y los fondos del Libro, la Música y el sector Audiovisual. 
ción cultural moderna no estar basada en las instituciones formales de la educación?

Es de esperar que una discusión en profundidad del proyecto de ley en el Congreso Nacional aborde éstas y otras preguntas.

\section{REFERENCIAS}

Benavente, J.M. \& J.J. Price. 2011. "Apoyo público a las artes y la cultura: Una mirada desde la economía”. En Cultura: oportunidad de desarrollo, editado por Magdalena Aninat. Santiago: Consejo Nacional de la Cultura y las Artes (CNCA).

Comisión Asesora Presidencial. 1991. Propuesta para la institucionalidad cultural chilena. Santiago: Ministerio de Educación.

Comisión Asesora Presidencial. 1997. Chile está en deuda con la cultura. Comisión asesora presidencial en materias artístico culturales. Santiago: Gobierno de Chile.

Comisión Asesora Presidencial. 2013. Institucionalidad. Ciencia, tecnología e innovación. Santiago: Gobierno de Chile.

Consejo Nacional de Innovación para la Competitividad (CNIC). 2007. Hacia una estrategia nacional de innovación. Libro Blanco. Vol. 1. Santiago: CNIC.

Consejo Nacional de la Cultura y las Artes. 2005. Chile quiere más cultura. Definiciones de política cultural 2005-2010. Valparaíso: CNCA.

- 2011. Politica cultural 2011-2016. Valparaíso: CNCA.

- 2012. Los Estados de la cultura. Estudio sobre la institucionalidad cultural pública de los países del SICSUR. Valparaíso: CNCA.

- 2014. Mapeo de las industrias creativas en Chile. Caracterización y dimensionamiento. Santiago: CNCA.

Chartrand, H. \& C. McCaughey. 1989. "The Arm's Lenght Principle and the Arts: An International Perspective-Past, Present and Future". En Who's to Pay for the Arts? The International Search for Models of Support, editado por M.C. Cummings \& J.M. Schuster. New York City: American Council for the Arts.

Garretón, M.A., J.M. Barbero, M. Cavarozzi, N. García Canclini, G. Ruiz-Giménez \& R. Stavenhagen. 2003. El espacio cultural latinoamericano. Bases para una política de integración. Santiago: Andrés Bello / Fondo de Cultura Económica.

O'Hagan, J.W. 1998. The State and the Arts: An Analysis of Key Economic Policy Issues in Europe and the United States. Cheltenham, UK, \& Northampton, Mass: Edward Elgar Publishing.

Palominos, S. 2014. Chile. World CP Profile. Sidney: IFACCA.

Price, J.J. 2011. How To Support the Culture and The Arts? Supply Subsidies versus Demand Vouchers. Tesis no publicada. London School of Economics. 
Robinson, J.P., C.A. Keegan, M. Karth \& T.A. Triplett. 1985. Public Participation in the Arts: Final Report on the 1982 Survey. Washington, D.C.: National Endowment for the Arts.

Sabel, Ch. 2012. "Self-Discovery as a Coordination Problem: Lessons from a Study of New Exports in Latin America". En Export Pioneers in Latin America, editado por Charles Sabel, Eduardo Fernández-Arias, Ricardo Hausmann, Andrés Rodríguez-Clare \& Ernesto Stein. Washington, D.C.: Inter-American Development Bank.

Schuster, J.M. 1991. "The Audience for American Art Museums". NEA Research Division Report No. 23. Santa Ana, California: Seven Locks Press.

Soto-Kloss, Eduardo. 2013. Informe en derecho preparado para el CNCA el año 2013.

Squella, A. 2006. "La nueva institucionalidad cultural". En La cultura durante el periodo de la transición a la democracia 1990-2005, editado por E. Carrasco \& B. Negrón. Valparaíso: CNCA.

- 2008. La nueva institucionalidad cultural de Chile. Valparaíso: Edeval.

Subercaseaux, B. 2006. "Cultura y democracia". En La cultura durante el periodo de la transición a la democracia 1990-2005, editado por E. Carrasco \& B. Negrón. Valparaíso: CNCA.

Throsby, D. 2008. "The Concentric Circles Model of the Cultural Industries". Cultural Trends 17 (3): 147-164.

- 2010. The Economics of Cultural Policy. Cambridge: Cambridge University Press.

Williamson, J. 1990. "What Washington Means by Political Reform”. En Latin American Adjustment: How Much Has Happened?, editado por J. Williamson. Washington: Institute of International Economics.

Williamson, O. 1964. The Economics of Discretionary Behaviour. Englewood Cliffs, N.J: Prentice-Hall. 


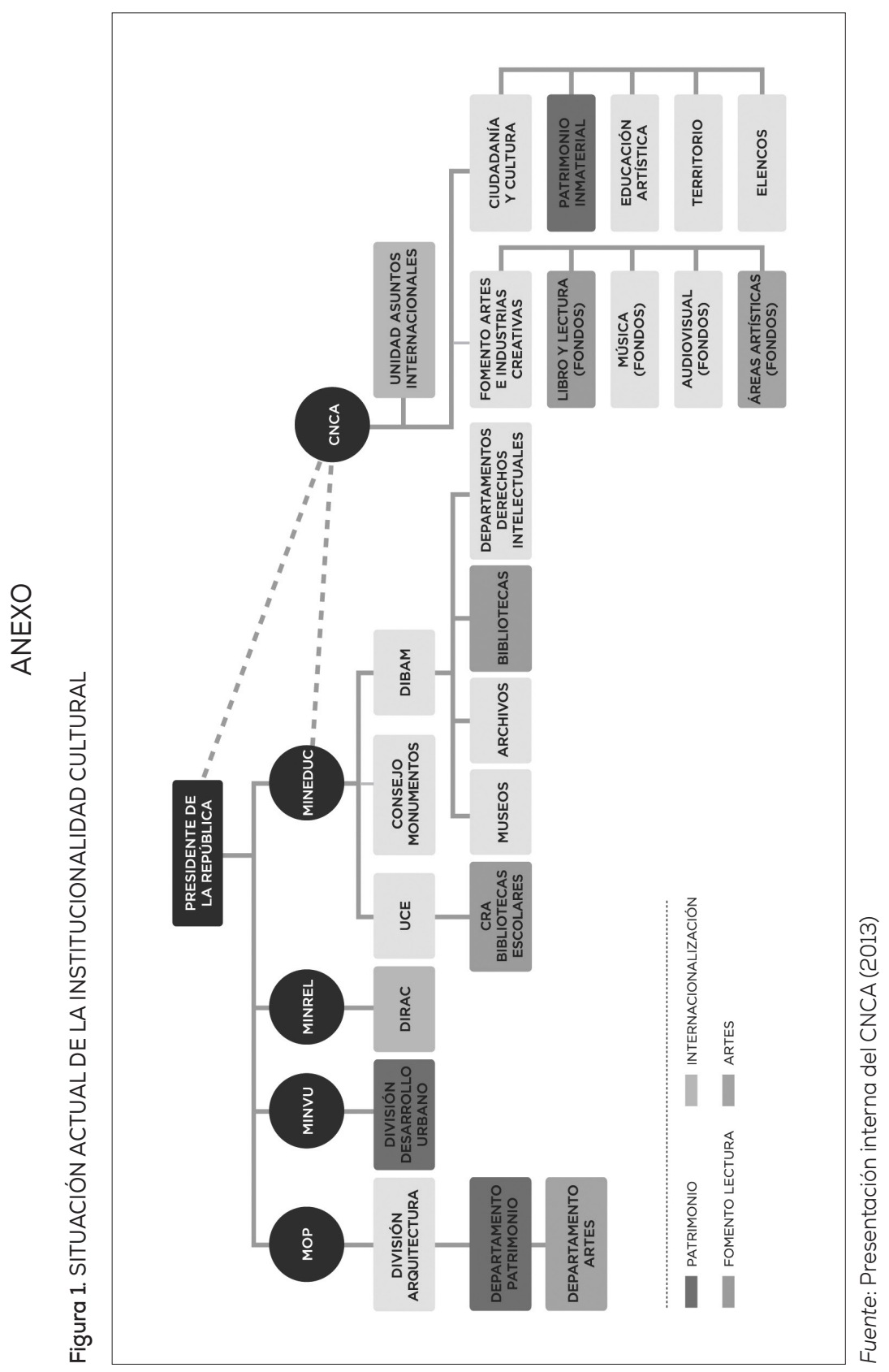




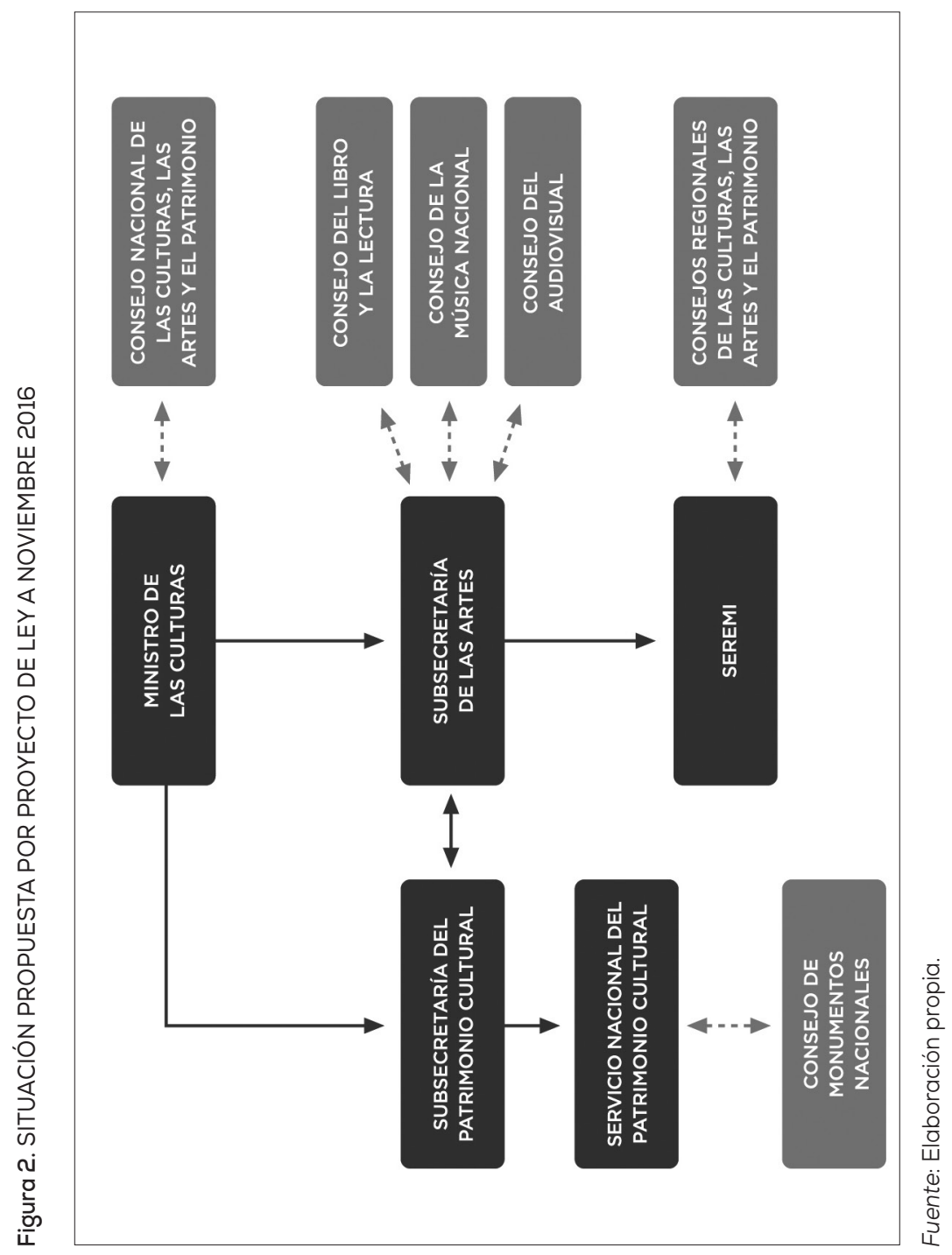



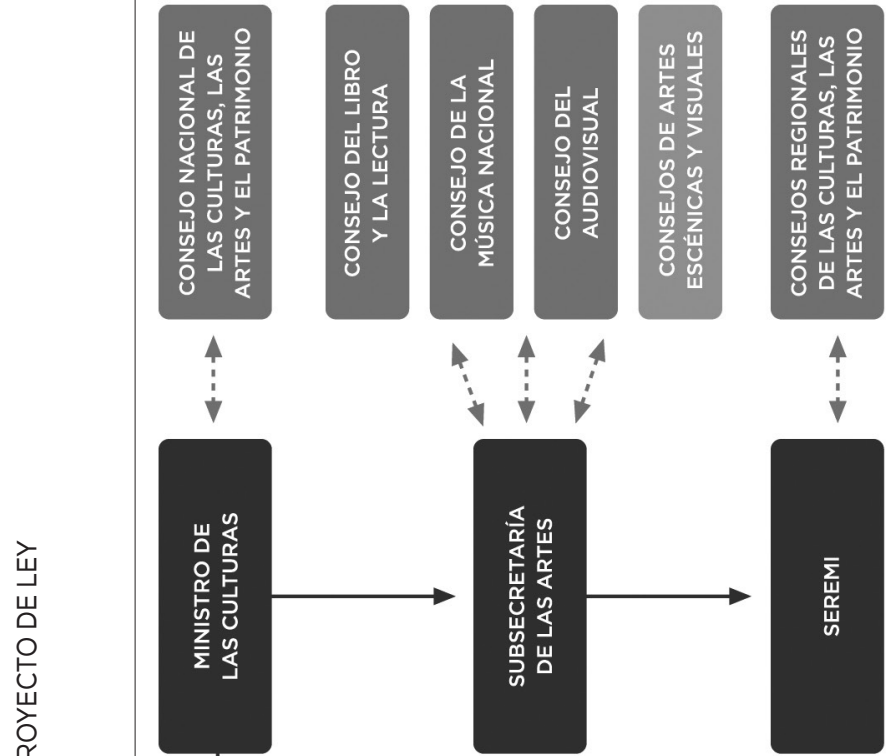

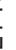

z

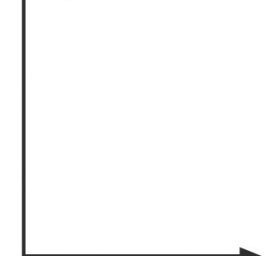

$\downarrow$

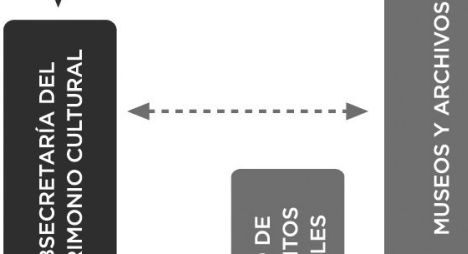

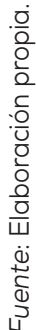

